\title{
Comparative transcriptomic analyses of powdery mildew resistant and susceptible cultivated cucumber (Cucumis sativus L.) varieties to identify the genes involved in the resistance to Sphaerotheca fuliginea infection
}

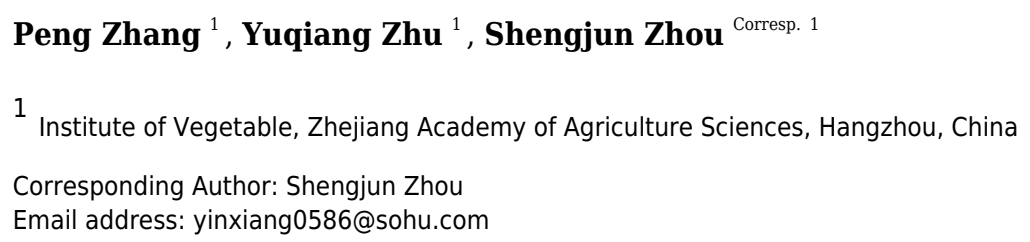

Background: Cucumber (Cucumis sativus L.) is a widely cultivated vegetable crop, and its yield and quality are greatly affected by various pathogen infections. Sphaerotheca fuliginea is a pathogen that causes powdery mildew (PM) disease in cucumber. However, the genes involved in the resistance to PM in cucumber are largely unknown. Methods: In our study, a cucumber PM resistant cultivated variety 'BK2' and a susceptible cultivated variety 'H136' were used to screen and identify differential expressed genes (DEGs) under the S. fuliginea infection. Results: There were only 97 DEGs between BK2 and H136 under the control condition, suggesting a similarity in the basal gene expression between the resistant and susceptible cultivated varieties. A large number of hormone signaling-related DEGs (9.2\% of all DEGs) between resistant and susceptible varieties were identified, suggesting an involvement of hormone signaling pathways in the resistance to PM. In our study, the defense-related DEGs belonging to Class I were only induced in susceptible cultivated variety and the defense-related DEGs belonging to Class II were only induced in resistant cultivated variety. The peroxidase, NBS, glucanase and chitinase genes that were grouped into Class I and II might contribute to production of the resistance to PM in resistant cultivated variety. Furthermore, several members of Pathogen Response-2 family, such as glucanases and chitinases, were identified as DEGs, suggesting that cucumber might enhance the resistance to PM by accelerating the degradation of the pathogen cell walls. Our data allowed us to identify and analyze more potential genes related to $\mathrm{PM}$ resistance. 
1 Comparative transcriptomic analyses of powdery mildew resistant and 2 susceptible cultivated cucumber (Cucumis sativus L.) varieties to identify the

3 genes involved in the resistance to Sphaerotheca fuliginea infection

4

5 Peng Zhang ${ }^{1}$, Yuqiang Zhu ${ }^{1}$ and Shengjun Zhou ${ }^{1 *}$

6

$7{ }^{1}$ Institute of Vegetable, Zhejiang Academy of Agriculture Sciences, Hangzhou, China

8

$9 *$ Corresponding author:

10 Shengjun Zhou $^{1}$

11 Desheng Zhong Road, 298\#, Hangzhou, Zhejiang Province, 310021, China

12 Email address: yinxiang0586@sohu.com

13

14

15 


\section{Abstract}

32 Background:

33 Cucumber (Cucumis sativus L.) is a widely cultivated vegetable crop, and its yield and quality 34 are greatly affected by various pathogen infections. Sphaerotheca fuliginea is a pathogen that 35 causes powdery mildew (PM) disease in cucumber. However, the genes involved in the 36 resistance to PM in cucumber are largely unknown.

37 Methods:

38 In our study, a cucumber PM resistant cultivated variety 'BK2' and a susceptible cultivated 39 variety 'H136' were used to screen and identify differential expressed genes (DEGs) under the $S$. 40 fuliginea infection.

41 Results:

42 There were only 97 DEGs between BK2 and H136 under the control condition, suggesting a 43 similarity in the basal gene expression between the resistant and susceptible cultivated varieties. 44 A large number of hormone signaling-related DEGs (9.2\% of all DEGs) between resistant and 45 susceptible varieties were identified, suggesting an involvement of hormone signaling pathways 46 in the resistance to PM. In our study, the defense-related DEGs belonging to Class I were only 47 induced in susceptible cultivated variety and the defense-related DEGs belonging to Class II 48 were only induced in resistant cultivated variety. The peroxidase, NBS, glucanase and chitinase 49 50 genes that were grouped into Class I and II might contribute to production of the resistance to PM in resistant cultivated variety. Furthermore, several members of Pathogen Response-2 family, such as glucanases and chitinases, were identified as DEGs, suggesting that cucumber might enhance the resistance to PM by accelerating the degradation of the pathogen cell walls. Our data allowed us to identify and analyze more potential genes related to PM resistance. 


\section{Introduction}

63 Cucumber (Cucumis sativus L.) is an economically important vegetable crop cultivated 64 worldwide, and its production is affected by a variety of infective agents (Zhang et al., 2008). 65 Powdery mildew (PM) caused by Sphaerotheca fuliginea is the most destructive disease in 66 cucumber (Zhang et al., 2012). S. fuliginea is a pathogen with a narrow host range limited to the 67 Cucurbitaceae (Savory et al., 2011). Recently, massive data on the epidemiology, host specificity, and genome of $S$. fuliginea have been studied and published (Arauz et al., 2010; Tian et al., 2011). P. fuliginea infects the host plants either by conidia or ascospores. Once the conidia have touched the host surface for a few hours, the germ tube and appressorium emerges from the conidium and penetrates the host cell wall (Huckelhoven, 2005). After the barrier id defeated, the infection structures are developed soon (Kavkova and Curn, 2005). The pathogen has a broad host range and can survive on a variety of cucurbit crops during the growing seasons (He et al., 2013). PM infects foliage and causes a reduction in photosynthetic activity at the early stage of cucumber development (Oerke et al., 2006). The rapid infection process of $S$. fuliginea makes PM difficult to be controlled in nature (Olczak-Woltman et al., 2011).

Selective breeding for PM-resistant cucumber varieties is an environmentally friendly and effective way to control the disease (Mcgrath, 2001). Several PM-resistant cucumber varieties, including classic PI197088 line, European greenhouse type S06, inbred line WI 2757, BK2, have been screened in the past years (He et al., 2013; Liu et al., 2008; Sakata et al., 2006; Zhang et al., 2015). These PM-resistant varieties provided good materials for QTL mapping of PM resistance (Wang et al., 2018). During evolution, plants developed a two-level innate immune system against pathogen attacks (Jones and Dangl, 2006). The first level is preventing pathogens from entering the cell through pathogen-associated molecular pattern-triggered immunity (Zipfel and Robatzek, 2010). The second level consists of a number of resistance genes and proteins, which trigger the programmed cell death of pathogens (Cheng et al., 2012). The involvement of genes in PM resistance is complex, and no undisputed mechanisms have been determined (Liu et al., 2017).

PM-associated resistance genes have been screened and utilized. For example, in cucumber, a potential nucleotide-binding site (NBS)-containing, protein-encoding resistance gene analog, CSRGA23, is involved in the active defense response to pathogen infection (Wan et al., 2010). The CsERF004 expression pattern in resistant and susceptible cucumber cultivars indicated that 
93 CsERF004 plays a role in the resistance to pathogen infection (Liu et al., 2017). Additionally, 15

94 NBS-containing disease resistance genes have been isolated from the cucumber genome using 95 degenerate PCR primers (Ding et al., 2005). Owing to its limited genetic diversity, there have 96 been few advances in breeding for disease resistance in cucumber.

97 Several phytohormones, such as auxin, jasmonic acid (JA), salicylic acid (SA), and abscisic 98 acid (ABA), are involved in plant responses to pathogen infection (Jiang et al., 2010; Riemann et al., 2013; Takatsuji et al., 2012). Variations in phytohormone accumulation profiles among Vitis

100

101

102

103

104

105

106

107

108

109

110

111

112

113

114

115

116

117

118

119

120

121

122

123 cultivars contributed to PM resistance (Yin et al., 2017). Furthermore, species-specific transcriptional responses of hormone signaling genes to PM were discovered in various grapevine species (Polesani et al., 2010). Hormone-related genes may be involved in the resistance to PM in cucumber. Additionally, glucanases are abundant in plants and may be associated with resistance to phytopathogenic fungi (Johnson George et al., 2016). Glucanase genes are always co-expressed with other anti-pathogen genes, including chitinase and peroxidase, in various plant species (Balasubramanian et al., 2012).

Next-generation sequencing is a cost-efficient way to analyze transcriptomic variations under different treatment conditions (Shen et al., 2017; Yu et al., 2017). In 2012, expression profiling of $P$. cubensis-infected cucumber identified differential expressed genes (DEGs) during the infection process from 1 to $8 \mathrm{~d}$ post inoculation (Adhikari et al., 2012). Additionally, a $P$. cubensis transcriptome isolated during cucumber infection captured 2,383 P. cubensis DEGs in sporangia (Savory et al., 2012). The identification of the DEGs between resistant and susceptible cucumber varieties aids in the screening of candidate resistance genes and suggests a complex regulatory network in the plant-pathogen system (Burkhardt and Day, 2016). In our study, two cultivated cucumber varieties were used for RNA-seq, allowing us to identify and analyze more potential genes related to PM resistance.

\section{Materials \& Methods}

\section{Plant material and sampling}

A cucumber PM-resistant cultivated cucumber variety 'BK2' and a PM-susceptible cultivated variety 'H136' were used in our study (Zhang et al., 2015). Cucumber seedlings were planted in a greenhouse with a photoperiod of $12 \mathrm{~h}$ light $/ 12 \mathrm{~h}$ dark, relative humidity of $60 \%$, and light intensity of $120 \mu \mathrm{mol} \mathrm{m} \mathrm{m}^{-2} \mathrm{~s}^{-1}$. The third leaves of the seedlings at the three-leaf stage were 
124 sprayed with $S$. fuliginea for inoculation. The infected morphological results are showed in 125 Figure S1. A solution containing $S$. fuliginea sporangia $\left(2 \times 10^{6}\right.$ sporangia $\left./ \mathrm{mL}\right), 5 \mathrm{mM}$ glucose, 126 and $2.5 \mathrm{mM} \mathrm{KH} \mathrm{PO}_{4}$ was prepared as the inoculant. The seedlings sprayed with sterile water 127 were used as the controls. Both inoculated and control seedlings were kept in dark for $24 \mathrm{~h}$ and 128 separately covered with plastic film. In total, 10 seedlings per replicate and three replicates per 129 treatment group were tested. In our study, four independent cDNA library groups were constructed for Illumina RNA sequencing. Two library groups (three libraries for each group) were from a PM-resistant cultivated cucumber variety, including a water treated- and a $S$. fuliginea treated-materials. Two other library groups (three libraries for each group) were from a PM-susceptible cultivated cucumber variety, including a water treated- and a S. fuliginea treatedmaterials. Plant samples for each group were harvested at $48 \mathrm{~h}$ post inoculation and were immediately frozen in liquid $\mathrm{N}_{2}$ until use.

136

\section{RNA extraction and cDNA library preparation}

Total RNA was extracted using a TRIzol Kit according to its protocol (Promega, Beijing, China). There are three biological replications have been performed for each RNA groups. DNA contamination was removed by RNase-free DNase I (TaKaRa, Dalian, China). RNA concentrations were quantified using a NanoDrop ND-1000 spectrophotometer at $260 \mathrm{~nm}$ and $280 \mathrm{~nm}$. Three independent cDNA libraries for each sample group were constructed. The library of sensitive cultivated variety sprayed with sterile water was named 'SC', the library of sensitive cultivated variety sprayed with $S$. fuliginea named 'ST', the library of resistant cultivated variety sprayed with sterile water was named ' $\mathrm{RC}$ ', and the library of resistant cultivated variety sprayed with $S$. fuliginea named 'RT'. The four libraries were used for the transcriptome sequencing, separately, using Illumina HiSeqTM 4000 platform by Gene Denovo Co. (Guangzhou, China).

Raw reads were generated in a paired-end format with average length of $300 \mathrm{bp}$. The raw sequence data has been submitted to the NCBI Short Read Archive with accession numbers SRP212890 (https://www.ncbi.nlm.nih.gov/sra/SRP212890).

\section{Processing of RNA-seq data}

153 Raw data (raw reads) of fastq format were firstly processed through in-house perl scripts with average length of $300 \mathrm{bp}$. In this step, clean data (clean reads) were obtained by removing reads 
155 with the length less than $100 \mathrm{bp}$, and low quality reads from raw data. For reads quality control, 156 the reads with base quality $<10$ in more than $80 \%$ of reads, reads containing adapters, and reads 157 with an $\mathrm{N}$ ratio $>5 \%$ were considered to be low quality reads. At the same time, Q20, Q30, GC158 content and sequence duplication level of the clean data were calculated. All the downstream 159 analyses were based on clean data with high quality.

160

161 Comparative analysis and gene functional annotation

162 The adaptor sequences and low-quality sequence reads were removed from the data sets. These 163 clean reads were then mapped to the reference genome sequence (NCBI: ASM407v2). Only 164 reads without mismatch or with one mismatch were further analyzed and annotated based on the 165 reference genome. Tophat 2 tools software were used to map with reference genome. Gene 166 function was annotated based on the following databases: $\mathrm{Nr}$ (NCBI non-redundant protein 167 sequences, https://www.ncbi.nlm.nih.gov); Nt (NCBI non-redundant nucleotide sequences); 168 Pfam (Protein family, http://pfam.xfam.org/); KOG/COG (Clusters of Orthologous Groups of 169 proteins, http://clovr.org/docs/clusters-of-orthologous-groups-cogs/); Swiss-Prot (A manually 170 annotated and reviewed protein sequence database, https://web.expasy.org/docs/swiss171 prot_guideline.html); KO (KEGG Ortholog database, https://www.kegg.jp/); and GO (Gene 172 Ontology, https://www.ebi.ac.uk/ols/ontologies/go) (Hao et al., 2017). For functional annotation, 173 the unigenes, though a BLASTall algorithm-based program with a threshold of $E$-value $<10^{-10}$, 174 were searched against different databases.

\section{Quantification of gene expression levels}

177 The number of reads mapped to each unigene was counted using HTSeq software. 178 Quantification of gene expression levels were estimated by fragments per kilobase of transcript 179 per million fragments mapped (FPKM) method.

180

\section{Differential expression analysis}

182 Differential expression analysis of two groups was performed using the DESeq R package 183 (1.10.1). DESeq provide statistical routines for determining differential expression in digital gene expression data using a model based on the negative binomial distribution. The resulting $P$ 185 values were adjusted using the Benjamini and Hochberg's approach for controlling the false 
186 discovery rate. The differential expressed genes (DEGs) were screened with criterions: |log2 fold 187 change $\mid>1$ and statistical significance of adjusted $p$ value $<0.05$ (Anders and Huber, 2010).

188

189

190

191

192

193

194

195

196

197

198

199

200

201

202

203

204

205

206

207

208

209

210

211

212

213

214

215

216

\section{Enrichment analysis}

Gene Ontology (GO) enrichment analysis of the differentially expressed genes (DEGs) was implemented by the GOseq $\mathrm{R}$ packages based Wallenius non-central hyper-geometric distribution, which can adjust for gene length bias in DEGs.

KEGG is a database resource for understanding high-level functions and utilities of the biological system, such as the cell, the organism and the ecosystem, from molecular-level information, especially large-scale molecular datasets generated by genome sequencing and other high-throughput experimental technologies (http://www.genome.jp/kegg/). We used KOBAS software to test the statistical enrichment of differential expression genes in KEGG pathways (Xie et al., 2011). A two-tailed Fisher's exact test was used to analyze the GO and KEGG functional enrichments. GO and KEGG categories with a corrected $P$ value $<0.05$ was considered significant.

\section{K-means cluster}

ClusGap R function-cluster package (v.2.0.5) was used to determine the optimal number of clusters. Subsequently, K-means clustering with default algorithm was used to get clusters using the scaled normalized relative gene expression data on a $\log 2$ scale for each expressed gene. The results of clustering were displayed using $\mathrm{MeV}$ program.

\section{Real-time PCR validation}

Total RNAs from different sample groups were isolated by a Plant RNeasy Mini kit (Qiagen, Hilden, Germany) according to its protocol. QRT-PCR was performed using the SYBR Premix Ex Taq Kit (TaKaRa, Dalian, China) and a DNA Sequence Detection System (ABI PRISM 7700, Applied Biosystems, Shanghai, China). Three independent samples from each group were used for the RT-PCR experiment. The EFla gene (accession number EF446145) was used as the internal standard gene to calculate relative fold differences by the values of comparative cycle threshold $\left(2^{-\Delta \Delta \mathrm{Ct}}\right)$. Sterile $\mathrm{ddH} 2 \mathrm{O}$ was used as no-template control. In detail, $1 \mu \mathrm{L}$ cDNA was added to $5 \mu \mathrm{L}$ of $2 \times \mathrm{SYBR}^{\circledR}$ Green buffer, $0.1 \mu \mathrm{M}$ of each primer and $\mathrm{ddH}_{2} \mathrm{O}$ to a working 
217 volume of $10 \mu \mathrm{L}$. The PCR program was $95^{\circ} \mathrm{C}$ for $10 \mathrm{~min}$, followed by 40 cycles of $95{ }^{\circ} \mathrm{C}$ for $21810 \mathrm{~s}$ and $60{ }^{\circ} \mathrm{C}$ for $60 \mathrm{~s}, 72{ }^{\circ} \mathrm{C}$ for $15 \mathrm{~s}$ at last.

219

220

\section{Statistical analysis}

Statistical data were analyzed using Statistical Program for Social Sciences (SPSS) 19.0 222 software (SPSS, Chicago, IL, USA). All of the expression analyses were performed for three

223 224 225 226 227 228 229 230 231

232

233

234

235

236

237

238

239

240

241

242

243

244

245

246

247 biological replicates. All reported values represent the averages of three replicates, and data are expressed as the mean plus or minus the standard deviation (mean $\pm \mathrm{SD}$ ).

\section{Results}

\section{Sequencing and sequence annotation}

In our study, a large number of raw reads were filtered to produce $\sim 280$ million clean reads (Table S1). Most of the clean reads could be mapped onto the reference genome (NCBI: ASM407v2), including 87.1\% unique mapped reads and 1.8\% multiple mapped reads (Table S2).

For gene annotation, the genes were queried against several databases, including $\mathrm{Nr}(21,343$ genes), SwissProt (20,889 genes), KEGG (7,556 genes), COG (8,024 genes), Pfam (17,235 genes), eggNOG $(20,239$ genes $)$ and KOG $(11,386)$. In total, 21,408 genes could be annotated to at least one database (Table S3). Most of the genes (11,386 genes) could be assigned to 25 functional KOG classifications. The largest KOG term, 'General function prediction only', contained 2,367 genes. 'Posttranslational modification, protein turnover, chaperones' (1,330 genes), 'Signal transduction mechanisms' (1,101 genes), 'translation' (771 genes), and 'Carbohydrate transport and metabolism' (718 genes) contained large numbers of the identified genes. Only four unigenes were classified into 'Cell motility' (Fig. 1). The detailed information of all identified unigenes, including gene id, functional description, ontology assignment, expression value, $p$-value, are shown in Table $\mathbf{S 4}$.

\section{GO and KEGG classifications of all identified genes}

In our study, most of the identified genes could be assigned to various functional terms belonging to the three major GO categories: biological process, cellular component, and molecular function. For biological process, 'metabolic processes', ‘single-organism process', and 
248 'cellular processes' were the dominant GO terms. For cellular component, the dominant terms 249 were 'cell', 'cell part', and 'organelle', and for molecular function, a large percentage of genes 250 were associated with 'catalytic activity' and 'binding' (Figure S2 and Table S5).

251 Furthermore, all of the unigenes mapped to canonical pathways in the KEGG database. In 252 total, 7,756 genes from cucumber were assigned to 126 KEGG pathways (Table S6). The largest 253 KEGG term was "ribosome" with 293 genes (ko03010). Additionally, 278 genes were associated 254 with the KEGG pathway "plant hormone signal transduction", 215 with "biosynthesis of amino 255 acids", and 211 with "carbon metabolism".

256

257

258

259

260

261

262

263

264

265

266

267 268

269

270

271

272

273

274

275

276

277 278

\section{Analysis of DEGs during $S$. fuliginea infections}

To compare the DEGs between different sample groups, expression profiles of the DEGs were displayed in a heatmap (Fig. 2a). A total of 3,410 DEGs were identified, including 2,312 DEGs in the ST vs RT comparison, 1,174 DEGs in the ST vs SC comparison, 1,044 DEGs in the RT vs RC comparison, and 97 DEGs in the SC vs RC comparison (Fig. 2b). To reflect the changing trends among sample groups, all of the DEGs were assigned into 12 clusters using MeV software by the K-means method. The genes belonging to the Clusters 1, 3, and 10 showed the highest expression levels in the RT sample group. The genes belonging to the Clusters 7 and 12 showed the highest expression levels in the ST sample group. The genes belonging to Clusters 4 and 8 showed the highest expression levels in the SC sample group, and the genes belonging to Clusters 9 and 11 showed the highest expression levels in the RC sample group (Fig. 2c).

\section{Analysis of the DEGs between resistant and susceptible cucumber varieties during $S$.} fuliginea infection

A GO enrichment analysis showed that several stress response-related GO terms were enriched under the $S$. fuliginea infection in the resistant variety. In detail, seven stress responserelated GO terms, including 'response to high light intensity', 'response to abscisic acid', 'response to reactive oxygen species', 'defense response to bacterium', 'response to jasmonic acid', 'response to inorganic substance' and 'response to nematode' terms, were significantly enriched in the RT vs RC comparison (Table S7). Only two stress response-related GO terms, including 'response to auxin' and 'response to abscisic acid' terms, were significantly enriched in the ST vs SC comparison (Table S8). 
279

280

281

282

283

284

285

286

287

288

289

290

291

292

293

294

295

296

297

298

299

300

301

302

303

304

305

306

307

308

309

A KEGG enrichment analysis showed that the expression levels of 21 "plant hormone signal transduction'-related genes were altered by the $S$. fuliginea infection in the resistant variety, while only 12 were altered in the susceptible variety. Moreover, the expression levels of 10 and 7 'plant-pathogen interaction'-related genes were altered by the $S$. fuliginea infection in the resistant and susceptible varieties, respectively. The number of metabolism-related genes that were altered by the $S$. fuliginea infection in the resistant variety was different from that in the susceptible variety. For example, the expression levels of seven 'flavonoid biosynthesis'-related genes, such as F3M (Csa1G013190), CHS2 (Csa3G600020), CYP98A44 (Csa3G819790), CHI (Csa5G505170), F3H (Csa6G108510), CYP73A11(Csa6G133710) and HST (Csa7G431440), were altered in the resistant variety but only three genes, such as CYP98A44 (Csa3G819790), FLS (Csa6G040540) and HST (Csa7G431440), were altered in the susceptible variety. In addition, the expression levels of 14 'starch and sucrose metabolism'-related genes were altered in the resistant variety, while 21 were altered in the susceptible variety (Fig. 3). The significance $P$ values of each KEGG pathway in the ST vs SC and RT vs RC comparisons are shown in Table S9 and S10.

Furthermore, the DEGs in the RT vs ST comparison were analyzed, and the top 20 enriched KEGG terms are shown in Fig. 4a. In total, 154 DEGs were classified into the 'ribosome' term, 49 DEGs to the 'plant hormone signal transduction' term, 27 DEGs to the 'starch and sucrose metabolism' term, 19 DEGs to the 'biosynthesis of amino acids' term, and 19 DEGs to the 'carbon metabolism' term.

\section{The involvement of hormone signaling in PM resistance}

In our study, a large number of hormone-related DEGs $(9.2 \%$ of all DEGs) were identified between the resistant and susceptible cucumber varieties during $S$. fuliginea infection. The KEGG analysis assigned most of the DEGs to key components involved in various hormonal signaling pathways, including those of auxin, cytokinin, gibberellin, ABA, brassinosteroid, JA and SA (Fig. 4b). For the auxin signaling pathway, one $A U X 1$ (K13946) gene, one GH3 (K14487) gene, one $A U X / I A A$ gene (K14484) and ten $S A U R$ (K14488) genes were significantly down-regulated and two $A U X / I A A$ genes and one $S A U R$ gene were significantly up-regulated in ST compared with in RT. For the cytokinin signaling pathway, two AHP (K14490) genes were significantly up-regulated and three $A-A R R$ (K14492) genes were down-regulated in ST 
310 compared with in RT. For the gibberellin signaling pathway, one GID1 (K14493) gene was 311 significantly up-regulated and one GID2 (K14495) gene was significantly down-regulated in ST 312 compared with in RT. For the ABA signaling pathway, two PYR/PYL (K14496) genes, five 313 PP2C (K14497) genes, two SnRK2 (K14498) genes, and one $A B F$ (K14432) gene were 314 significantly up-regulated and four PYR/PYL (K14496) genes were significantly down-regulated 315 in ST compared with in RT. For the brassinosteroid signaling pathway, one BAK1 (K14506) 316 gene and one $B S K(\mathrm{~K} 14500)$ gene were significantly up-regulated and two CYCD3 (K14505) 317 genes were significantly down-regulated in ST compared with in RT. For the JA signaling 318 pathway, one JARl (K14506) gene and one JAZ (K13464) gene were significantly up-regulated 319 and one $J A Z$ gene was significantly down-regulated in ST compared with in RT. For the SA 320 signaling pathway, two TGA (K14431) genes and one PR-1 (K13449) gene were significantly 321 up-regulated in ST compared with in RT.

322

323

324

325 326

327

328

329

330

331

332

333

334

335

336

337

338

339

\section{Identification of classical defense-related DEGs}

In our study, a large number of defense-related genes were identified as DEGs during the $S$. fuliginea infection. In total, 49 NBS genes, 62 glucanase genes, 36 chitinase genes, and 152 peroxidase genes were identified in cucumber. Among the NBS-containing genes, 5 significantly up-regulated (Class I) and 14 significantly down-regulated (Class II) genes were identified. Among the glucanase genes, seven significantly up-regulated (Class I) and eight significantly down-regulated (Class II) genes were identified. Among the chitinase genes, 6 significantly upregulated (Class I) and 15 significantly down-regulated (Class II) genes were identified, and among the peroxidase genes, 42 significantly up-regulated (Class I) and 19 significantly downregulated (Class II) genes were identified (Fig. 5).

\section{Validation of the expression levels of several key genes}

To verify the changes in the expression levels of several important hormone- and defenserelated genes, a qRT-PCR assay with independent samples was performed. In total, eight genes, including four hormone-related genes and four defense-related genes, were randomly selected to check the RNA-seq data (Fig. 6). Our qRT-PCR results basically confirmed the RNA-seq data. All the primer sequences are listed in Table S11. 


\section{Discussion}

342 The molecular and genetic mechanisms associated with host resistance to PM have been

343 investigated to a limited extent in cucumber. Understanding the genes that are responsive to PM

344 will help reveal the regulatory mechanism underlying PM resistance. In 2011, a transcriptomic 345 study identified 87 DEGs associated with resistance to the PM pathogen in cucumber (Li et al., 346 2011). In 2012, up to 3,286 genes were differentially expressed in pair-wise comparisons 347 between different post-inoculation time points (Adhikari et al., 2012). In our study, two 348 cultivated cucumber varieties were used for RNA-seq, and they revealed variety-specific 349 transcriptional responses to PM.

350 The comparison of basal gene expression levels in resistant and susceptible cultivated 351 varieties under the control condition revealed only 86 DEGs, suggesting tiny variations for most 352 of the identified genes (Fig. 2b). During S. fuliginea infection, substantial variations in the 353 expression levels of thousands of genes were observed between resistant and susceptible 354 cultivated cucumber varieties. A large number of plant hormone signal transduction-related 355 genes were identified as DEGs in the ST vs RT comparison. Changes in phytohormone accumulation profiles differed between resistant and susceptible Vitis amurensis cultivars, suggesting the involvement of hormones in PM resistance (Yin et al., 2017). For example, grape PM resistance is affected by various hormones, including ethylene, JA, SA, and ABA (Denance et al., 2013). In our study, the genes encoding PP2C, SnRK2 and ABF were significantly affected by the $S$. fuliginea infection, suggesting an involvement of ABA signaling pathway in PM resistance. JAR1-mediated JA signaling pathway plays important roles in activating plant defenses (Kang et al., 2006). In cucumber, expression of the genes encoding JAR1 was significantly changed during the $S$. fuliginea infection, indicating the presence of jasmonic acidisoleucine-mediated defenses against $S$. fuliginea. Our data provide comprehensive information on hormone signaling pathway genes under $S$. fuliginea infection in cucumber.

The roles of auxin during interactions between plant pathogens and their hosts have been studied (Hussain et al., 2017). Many plant pathogen infections elevate free IAA levels or enhance auxin signaling (Kunkel and Harper, 2018). In cucumber, microRNA160b and its downstream target gene, AUXIN RESPOINSE FACTOR 16 (Csa6M445210.1), are responsive to pathogen infection (Jin and $\mathrm{Wu}, 2015$ ). During S. fuliginea infection, most auxin signaling 
$372 S A U R$ genes, were significantly expressed in the cultivated resistant variety, suggesting an 373 enhancement in auxin signaling during PM resistance. SA is another important hormone 374 involved in innate immunity (Huang et al., 2018). In soybean (Glycine max), GmMPK4-silenced 375 plants showed more resistance to PM and accumulated higher SA levels, suggesting a close 376 relationship between the SA level and the resistance to PM (Liu et al., 2011). In our study, two 377 TGA (Csa2G403160 and Csa6G031950) genes and one PR-1 (Cucumber_newGene_1053) gene 378 were significantly expressed in ST compared with in RT, indicating essential roles in PM 379 resistance in cucumber. In Arabidopsis, the JA signaling pathway is an intrinsic component 380 within the host's defense system (Zhang et al., 2017). The expression levels of several $M A P K$, $381 M A P K K$ and $M A P K K K$ genes were significantly affected by pathogen infections and JA 382 treatments (Wang et al., 2015). In our study, three key JA-related genes, one JAR1 and two JAZ 383 genes, were identified as DEGs between resistant and susceptible cultivated varieties, suggesting 384 a role for JA signaling in PM resistance. JAZ1 is essential for pathogen defense and wound

385 386 387 388 389 390 391 392 393 394 395 396 397 398 399 400 401 402 response (Suza and Staswick, 2008). In the resistant cucumber variety, the expression level of $J A Z 1$ (Csa3G119760) was higher than in the susceptible variety, which suggested that inhibiting JA signaling might contribute to PM resistance.

Resistance mechanisms involve numerous variations in biochemical and physiological functions during plant responses to pathogens (Taheri and Kakooee, 2017). A large number of defense-related genes have been revealed in model plants. For example, several cucumber NBScontaining genes are involved in the reorganization of cognate pathogen effector proteins and their perturbations (Bent and Mackey, 2007). Other defense-related genes, such as peroxidases, chitinases, and glucanases, may be associated with the initiation of resistance signaling after pathogen infection (Xue et al., 1998). In our study, the expression levels of a number of defenserelated genes were affected in both the resistant and susceptible cultivated cucumber varieties by S. fuliginea infection.

Furthermore, the generation and removal of reactive oxygen species (ROS) are affected by pathogen infection, and uncontrolled ROS accumulation enhances the susceptibility of plants to pathogens (Vellosillo et al., 2010). Peroxidases are involved in scavenging $\mathrm{ROS}$, such as $\mathrm{H}_{2} \mathrm{O}_{2}$, in infected plant cells (Barna et al., 2012). In our study, 42 peroxidase genes (Class I) were only induced in the susceptible cultivated variety, while 19 peroxidase genes (Class II) were only induced in the resistant cultivated variety (Fig. 5d). The differential expression of peroxidase 
403 genes might contribute to PM resistance. Glucanase genes, members of the PR-2 family, are 404 significantly up-regulated by pathogen inoculation and are involved in plant pathogen defense 405 (Su et al., 2016). Glucanases produced by plant hosts enhance the degradation of the oomycete 406 cell wall (York et al., 2004). Chitinases, members of the PR family, are responsible for the 407 hydrolysis of chitin, a major component of the cell walls of many fungal pathogens (Patel and 408 Goyal, 2017). In our study, the S. fuliginea infection induced more glucanase and chitinase genes 409 in the resistant cultivated variety than in the susceptible cultivated variety, suggesting that the 410 enhanced resistance to pathogens in cucumber might involve the accelerated degradation of the 411 pathogen's cell walls.

$412 P R$ genes are also up-regulated by the application of plant hormones, such as ethylene, JA, 413 and SA (Sels et al., 2008). During pathogen infections, activated hormone signaling promotes the 414 expression levels of $P R$ genes, which might play a role in pathogen resistance (Tang et al., 2017). 415 In our study, the $P R$ genes, such as PR2 (Csa3G099760), PR17 (Csa7G072790), PR1 416 (Csa1G420360) and PR4 (Csa2G010370), were identified as DEGs. Expression analysis showed 417 that SA-mediated stress- and pathogenesis-related defenses play an essential role in the responses 418 to $S$. fuliginea infection. Our data suggested that PM resistance was controlled by a complex 419 network consisting of various hormone signaling pathways.

420

421 Conclusions

422 Under $S$. fuliginea infection, there were great differences in the expression of genes related to 423 hormone signaling, peroxidase and cell wall degradation between the resistant and susceptible 424 cultivated varieties. Our data allowed us to identify and analyze more potential genes related to 425 PM resistance, accelerating the breeding for disease resistance in cucumber.

426

427 Acknowledgements

428 We are grateful to Prof. Kaidong Liu from Lingnan Normal University for technical support. 429 The authors declare that they have no competing interests.

430

431 References

432 Adhikari, B.N., Savory, E.A., Vaillancourt, B., Childs, K.L., Hamilton, J.P., Day, B., Buell, C.R., 433 2012. Expression Profiling of Cucumis sativus in Response to Infection by Pseudoperonospora 
434 cubensis. PloS one 7, e34954.

435 Anders, S., Huber, W., 2010. Differential expression analysis for sequence count data. Genome 436 biology 11, R106.

437 Arauz, L.F., Neufeld, K.N., Lloyd, A.L., Ojiambo, P.S., 2010. Quantitative models for 438 germination and infection of Pseudoperonospora cubensis in response to temperature and 439 duration of leaf wetness. Phytopathology 100, 959-967.

440 Balasubramanian, V., Vashisht, D., Cletus, J., Sakthivel, N., 2012. Plant $\beta$-1,3-glucanases: their 441 biological functions and transgenic expression against phytopathogenic fungi. Biotechnology 442 Letters 34, 1983-1990.

443 Barna, B., Fodor, J., Harrach, B.D., Pogany, M., Kiraly, Z., 2012. The Janus face of reactive 444 oxygen species in resistance and susceptibility of plants to necrotrophic and biotrophic pathogens. 445 Plant physiology and biochemistry : PPB 59, 37-43.

446 Bent, A.F., Mackey, D., 2007. Elicitors, effectors, and R genes: the new paradigm and a lifetime 447 supply of questions. Annual review of phytopathology 45, 399-436.

448 Burkhardt, A., Day, B., 2016. Transcriptome and Small RNAome Dynamics during a Resistant 449 and Susceptible Interaction between Cucumber and Downy Mildew. The Plant Genome 9.

450 Cheng, X., Tian, C.J., Li, A.N., Qiu, J.L., 2012. [Advances on molecular mechanisms of plant451 pathogen interactions]. Yi chuan $=$ Hereditas 34, 134-144.

452 Denance, N., Sanchez-Vallet, A., Goffner, D., Molina, A., 2013. Disease resistance or growth: 453 the role of plant hormones in balancing immune responses and fitness costs. Frontiers in plant 454 science 4, 155.

455 Ding, G., Qin, Z., Liu, H., Zhou, X., Chi, C., Zhikun, Wang, 2005. Analysis and Cloning of NBS 456 Class Disease Resistant Gene Analog in Cucumber. Acta Horticulturae Sinica.

457 Hao, J., Guo, H., Shi, X., Wang, Y., Wan, Q., Song, Y., Zhang, L., Dong, M., Shen, C., 2017. 458 Comparative proteomic analyses of two Taxus species (Taxus $\times$ media and Taxus mairei) reveals 459 variations in the metabolisms associated with paclitaxel and other metabolites. Plant and Cell 460 Physiology, pcx128-pcx128.

461 He, X., Li, Y., Pandey, S., Yandell, B.S., Pathak, M., Weng, Y., 2013. QTL mapping of powdery 462 mildew resistance in WI 2757 cucumber (Cucumis sativus L.). TAG. Theoretical and applied 463 genetics. Theoretische und angewandte Genetik 126, 2149-2161.

464 Huang, X.X., Zhu, G.Q., Liu, Q., Chen, L., Li, Y.J., Hou, B.K., 2018. Modulation of Plant 
465 Salicylic Acid-Associated Immune Responses via Glycosylation of Dihydroxybenzoic Acids. 466 Plant physiology 176, 3103-3119.

467 Huckelhoven, R., 2005. Powdery mildew susceptibility and biotrophic infection strategies. 468 FEMS microbiology letters 245, 9-17.

469 Hussain, A., Ullah, I., Hasnain, S., 2017. Microbial Manipulation of Auxins and Cytokinins in 470 Plants. Methods in molecular biology 1569, 61-72.

471 Jiang, C.J., Shimono, M., Sugano, S., Kojima, M., Yazawa, K., Yoshida, R., Inoue, H., Hayashi, 472 N., Sakakibara, H., Takatsuji, H., 2010. Abscisic Acid Interacts Antagonistically with Salicylic 473 Acid Signaling Pathway in Rice-Magnaporthe grisea Interaction. Molecular Plant-Microbe 474 Interactions 23, 791-798.

475 Jin, W., Wu, F., 2015. Identification and characterization of cucumber microRNAs in response to 476 Pseudoperonospora cubensis infection. Gene 569, 225-232.

477 Johnson George, K., Rosana Babu, O., Vijesh Kumar, I.P., Santhosh Eapen, J., Anandaraj, M., 478 2016. Interplay of genes in plant-pathogen interactions: In planta expression and docking studies 479 of a beta 1,3 glucanase gene from Piper colubrinum and a glucanase inhibitor gene from 480 Phytophthora capsici. Physiology and molecular biology of plants : an international journal of 481 functional plant biology 22, 567-573.

482 Jones, J.D., Dangl, J.L., 2006. The plant immune system. Nature 444, 323-329.

483 Kang, J.H., Wang, L., Giri, A., Baldwin, I.T., 2006. Silencing threonine deaminase and JAR4 in 484 Nicotiana attenuata impairs jasmonic acid-isoleucine-mediated defenses against Manduca sexta. 485 The Plant cell 18, 3303-3320.

486 Kavkova, M., Curn, V., 2005. Paecilomyces fumosoroseus (Deuteromycotina: Hyphomycetes) as 487 a potential mycoparasite on Sphaerotheca fuliginea (Ascomycotina: Erysiphales). 488 Mycopathologia 159, 53-63.

489 Kunkel, B.N., Harper, C.P., 2018. The roles of auxin during interactions between bacterial plant 490 pathogens and their hosts. Journal of experimental botany 69, 245.

491 Li, J.W., Liu, J., Zhang, H., Xie, C.H., 2011. Identification and transcriptional profiling of 492 differentially expressed genes associated with resistance to Pseudoperonospora cubensis in 493 cucumber. Plant cell reports 30, 345-357.

494 Liu, D., Xin, M., Zhou, X., Wang, C., Zhang, Y., Qin, Z., 2017. Expression and functional 495 analysis of the transcription factor-encoding Gene CsERF004 in cucumber during 
496 Pseudoperonospora cubensis and Corynespora cassiicola infection. BMC plant biology 17, 96.

497 Liu, J.Z., Horstman, H.D., Braun, E., Graham, M.A., Zhang, C., Navarre, D., Qiu, W.L., Lee, Y., 498 Nettleton, D., Hill, J.H., Whitham, S.A., 2011. Soybean homologs of MPK4 negatively regulate 499 defense responses and positively regulate growth and development. Plant physiology 157, 13635001378.

501 Liu, L., Cai, R., Yuan, X., He, H., Pan, J., 2008. QTL molecular marker location of powdery 502 mildew resistance in cucumber (Cucumis sativus L.). Science in China. Series C, Life sciences 503 51, 1003-1008.

504 Mcgrath, M.T., 2001. Fungicide resistance in cucurbit powdery mildew: experiences and 505 challenges. Plant Disease 85, 236-245.

506 Oerke, E.C., Steiner, U., Dehne, H.W., Lindenthal, M., 2006. Thermal imaging of cucumber 507 leaves affected by downy mildew and environmental conditions. Journal of experimental botany $50857,2121-2132$.

509 Olczak-Woltman, H., Marcinkowska, J., Niemirowicz-Szczytt, K., 2011. The genetic basis of 510 resistance to downy mildew in Cucumis spp.--latest developments and prospects. Journal of 511 Applied Genetics 52, 249-255.

512 Patel, S., Goyal, A., 2017. Chitin and chitinase: Role in pathogenicity, allergenicity and health. 513 International journal of biological macromolecules 97, 331-338.

514 Polesani, M., Bortesi, L., Ferrarini, A., Zamboni, A., Fasoli, M., Zadra, C., Lovato, A., Pezzotti, 515 M., Delledonne, M., Polverari, A., 2010. General and species-specific transcriptional responses 516 to downy mildew infection in a susceptible (Vitis vinifera) and a resistant (V. riparia) grapevine 517 species. BMC genomics 11, 117.

518 Riemann, M., Haga, K., Shimizu, T., Okada, K., Ando, S., Mochizuki, S., Nishizawa, Y., 519 Yamanouchi, U., Nick, P., Yano, M., 2013. Identification of rice Allene Oxide Cyclase mutants 520 and the function of jasmonate for defence against Magnaporthe oryzae. Plant Journal for Cell \& 521 Molecular Biology 74, 226-238.

522 Sakata, Y., Kubo, N., Morishita, M., Kitadani, E., Sugiyama, M., Hirai, M., 2006. QTL analysis 523 of powdery mildew resistance in cucumber (Cucumis sativus L.). TAG. Theoretical and applied 524 genetics. Theoretische und angewandte Genetik 112, 243-250.

525 Savory, E.A., Adhikari, B.N., Hamilton, J.P., Vaillancourt, B., Buell, C.R., Day, B., 2012. 526 mRNA-Seq Analysis of the Pseudoperonospora cubensis Transcriptome During Cucumber 
527 (Cucumis sativus L.) Infection. PloS one 7, e35796.

528 Savory, E.A., Granke, L.L., Quesada-Ocampo, L.M., Varbanova, M., Hausbeck, M.K., Day, B., 529 2011. The cucurbit downy mildew pathogen Pseudoperonospora cubensis. Molecular plant 530 pathology $12,217-226$.

531 Sels, J., Mathys, J., De Coninck, B.M., Cammue, B.P., De Bolle, M.F., 2008. Plant pathogenesis532 related (PR) proteins: a focus on PR peptides. Plant physiology and biochemistry : PPB 46, 941533950.

534 Shen, C., Guo, H., Chen, H., Shi, Y., Meng, Y., Lu, J., Feng, S., Wang, H., 2017. Identification 535 and analysis of genes associated with the synthesis of bioactive constituents in Dendrobium 536 officinale using RNA-Seq. Scientific reports 7, 187.

537 Su, Y., Wang, Z., Liu, F., Li, Z., Peng, Q., Guo, J., Xu, L., Que, Y., 2016. Isolation and 538 Characterization of ScGluD2, a New Sugarcane beta-1,3-Glucanase D Family Gene Induced by 539 Sporisorium scitamineum, ABA, $\mathrm{H} 2 \mathrm{O} 2, \mathrm{NaCl}$, and $\mathrm{CdCl} 2$ Stresses. Frontiers in plant science 7 , 5401348.

541 Suza, W.P., Staswick, P.E., 2008. The role of JAR1 in Jasmonoyl-L: -isoleucine production 542 during Arabidopsis wound response. Planta 227, 1221-1232.

543 Taheri, P., Kakooee, T., 2017. Reactive oxygen species accumulation and homeostasis are 544 involved in plant immunity to an opportunistic fungal pathogen. Journal of Plant Physiology 216, 545 152-163.

546 Takatsuji, H., Jiang, C.J., Sugano, S., 2012. Salicylic acid signaling pathway in rice and the 547 potential applications of its regulators. Japan Agricultural Research Quarterly 44, 217-223.

548 Tang, Y., Liu, Q., Liu, Y., Zhang, L., Ding, W., 2017. Overexpression of NtPR-Q Up-Regulates

549 Multiple Defense-Related Genes in Nicotiana tabacum and Enhances Plant Resistance to 550 Ralstonia solanacearum. Frontiers in plant science 8, 1963.

551 Tian, M., Win, J., Savory, E., Burkhardt, A., Held, M., Brandizzi, F., Day, B., 2011. 454

552 Genome sequencing of Pseudoperonospora cubensis reveals effector proteins with a QXLR 553 translocation motif. Molecular plant-microbe interactions : MPMI 24, 543-553.

554 Vellosillo, T., Vicente, J., Kulasekaran, S., Hamberg, M., Castresana, C., 2010. Emerging 555 complexity in reactive oxygen species production and signaling during the response of plants to 556 pathogens. Plant physiology 154, 444-448.

557 Wan, H., Zhao, Z., Malik, A.A., Qian, C., Chen, J., 2010. Identification and characterization of 
558 potential NBS-encoding resistance genes and induction kinetics of a putative candidate gene

559

560

561

562

563

564

565

566

567

568

569

570

57

572

573 Yin, L., Qu, J., Deng, S., Liu, S., Lu, J., Zhang, Y., 2017. Phytohor

575 York, W.S., Qin, Q., Rose, J.K., 2004. Proteinaceous inhibitors of endo-beta-glucanases. 576 Biochimica et biophysica acta 1696, 223-233.

577 Yu, C., Guo, H., Zhang, Y., Song, Y., Pi, E., Yu, C., Zhang, L., Dong, M., Zheng, B., Wang, H., 578 Shen, C., 2017. Identification of potential genes that contributed to the variation in the taxoid 579 580

581

582

583

584

585

586

587

588

associated with downy mildew resistance in Cucumis. BMC plant biology 10, 186.

Wang, J., Pan, C., Wang, Y., Ye, L., Wu, J., Chen, L., Zou, T., Lu, G., 2015. Genome-wide identification of MAPK, MAPKK, and MAPKKK gene families and transcriptional profiling analysis during development and stress response in cucumber. BMC genomics 16, 386.

Wang, Y., VandenLangenberg, K., Wen, C., Wehner, T.C., Weng, Y., 2018. QTL mapping of downy and powdery mildew resistances in PI 197088 cucumber with genotyping-by-sequencing in RIL population. TAG. Theoretical and applied genetics. Theoretische und angewandte Genetik 131, 597-611.

Xie, C., Mao, X., Huang, J., Ding, Y., Wu, J., Dong, S., Kong, L., Gao, G., Li, C.Y., Wei, L., 2011. KOBAS 2.0: a web server for annotation and identification of enriched pathways and diseases. Nucleic Acids Res 39, W316-322.

Xue, L., Charest, P.M., Jabaji-Hare, S.H., 1998. Systemic Induction of Peroxidases, 1,3-betaGlucanases, Chitinases, and Resistance in Bean Plants by Binucleate Rhizoctonia Species. Phytopathology 88, 359-365.

contents between two Taxus species (Taxus media and Taxus mairei). Tree Physiology 37, 16591671.

Zhang, M.Z., Ye, D., Wang, L.L., Pang, J.L., Zhang, Y.H., Zheng, K., Bian, H.W., Han, N., Pan, J.W., Wang, J.H., Zhu, M.Y., 2008. Overexpression of the cucumber LEAFY homolog CFL and hormone treatments alter flower development in gloxinia (Sinningia speciosa). Plant molecular biology 67, 419-427.

Zhang, P., Zhu, Y., Wang, L., Chen, L., Zhou, S., 2015. Mining candidate genes associated with powdery mildew resistance in cucumber via super-BSA by specific length amplified fragment (SLAF) sequencing. BMC genomics 16, 1058.

Zhang, W., Corwin, J.A., Copeland, D., Feusier, J., Eshbaugh, R., Chen, F., Atwell, S., 
589 Kliebenstein, D.J., 2017. Plastic Transcriptomes Stabilize Immunity to Pathogen Diversity: The 590 Jasmonic Acid and Salicylic Acid Networks within the Arabidopsis/Botrytis Pathosystem. The 591 Plant cell 29, 2727-2752.

592 Zhang, Y., Pu, Z., Qin, Z., Zhou, X., Liu, D., Dai, L., Wang, W., 2012. A Study on the 593 Overwintering of Cucumber Downy Mildew Oospores in China. Journal of Phytopathology 160, $594 \quad 469-474$.

595 Zipfel, C., Robatzek, S., 2010. Pathogen-associated molecular pattern-triggered immunity: veni, 596 vidi...? Plant physiology 154, 551-554.

597 


\section{Figure 1}

\section{Functional KOG classification of all annotated genes in cucumber. $t$}

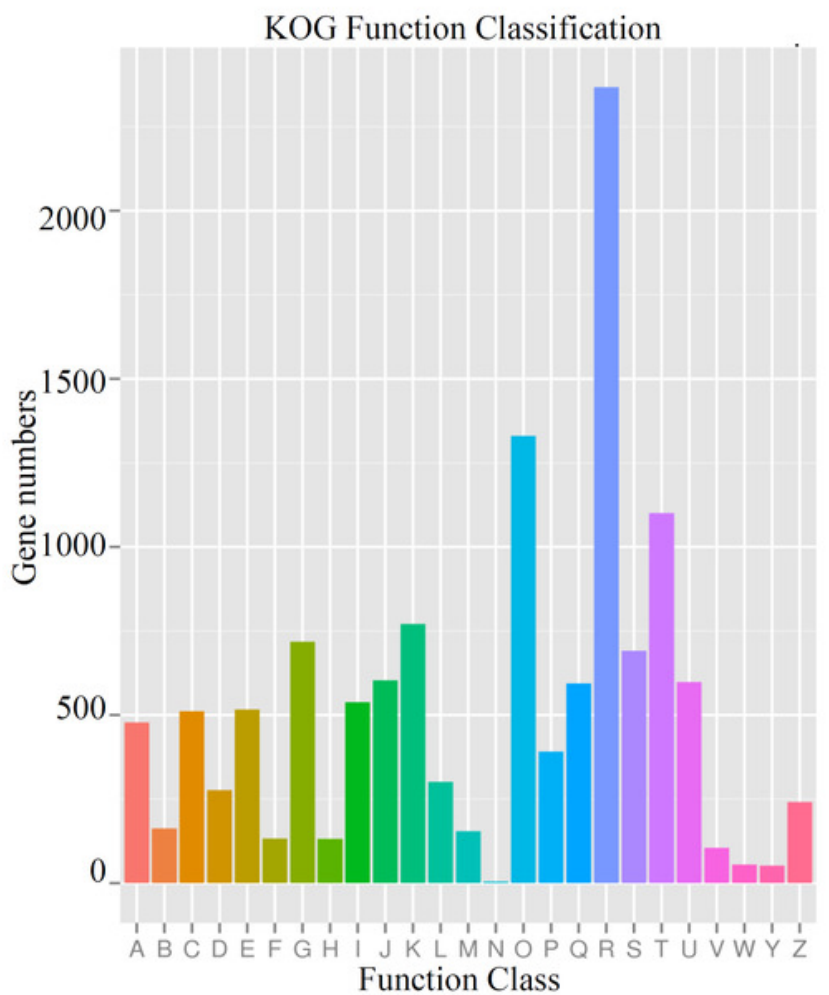

Function Class
A: RNA processing and modification (478 genes)

B: Chromatin structure and dynamics (163 genes)

C: Energy production and conversion (511 genes)

D: Cell cycle control, cell division, chromosome partitioning (276 genes)

E: Amino acid transport and metabolism (516 genes)

F: Nucleotide transport and metabolism (133 genes)

G: Carbohydrate transport and metabolism (718 genes)

$\mathrm{H}$ : Coenzyme transport and metabolism (132 genes)

I: Lipid transport and metabolism (538 genes)

$\mathrm{J}$ : Translation, ribosomal structure and biogenesis (603 genes)

$\mathrm{K}$ : Transcription (771 genes)

L: Replication, recombination and repair (301 genes)

M: Cell wall/membrane/envelope biogenesis (154 genes)

$\mathrm{N}$ : Cell motility (4 genes)

O:Posttranslational modification, protein turnover, chaperones (1330 genes)

P: Inorganic ion transport and metabolism (391 genes)

Q: Secondary metabolites biosynthesis, transport and catabolism (594 genes)

$\mathrm{R}$ : General function prediction only (2367 genes)

S: Function unknown (691 genes)

T: Signal transduction mechanisms (1101 genes)

U: Intracellular trafficking, secretion, and vesicular transport (598 genes)

V: Defense mechanisms (105 genes)

W: Extracellular structures ( 55 genes)

Y: Nuclear structure ( 52 genes)

Z: Cytoskeleton (241 genes) 
Figure 2

Expression profiles of the differentially expressed genes

(A) Heat map for cluster analysis of the differentially expressed unigenes by K-means

method. Yellow indicates up-regulated genes and blue indicates down-regulated genes. (B)

VennDiagrams of the DEGs in different comparisons.(C-N) MeV cluster analysis of

differentially expressed genes from the gene expression profiles. 
A

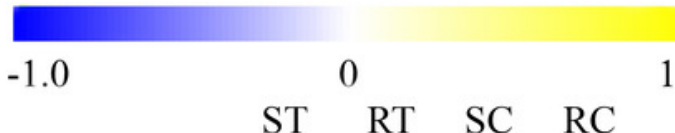

B
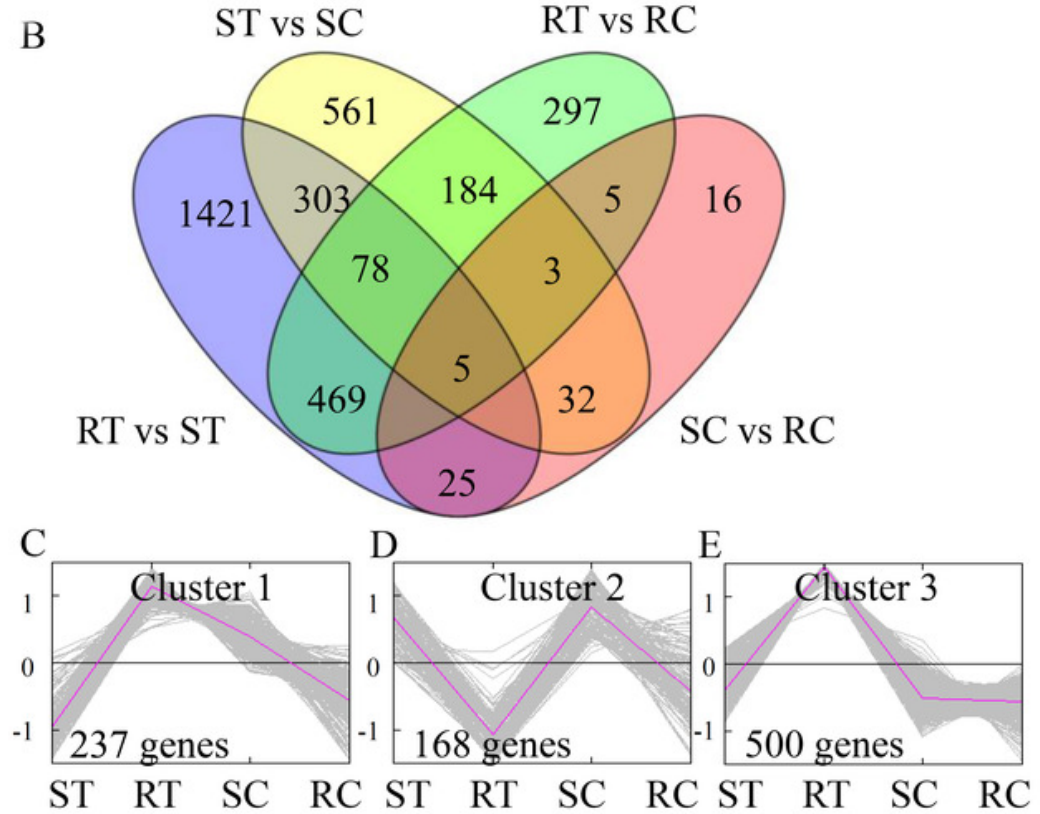
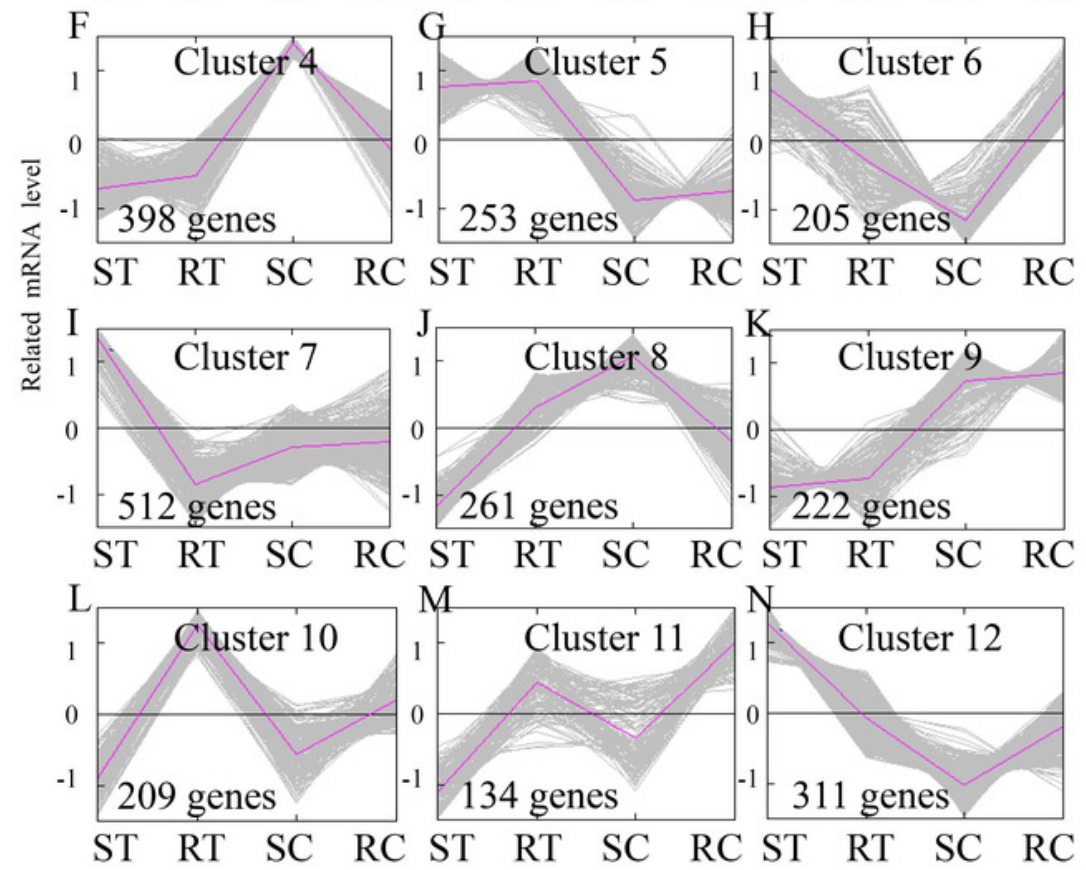
Identification of the DEGs in different comparisons.

\section{(a) Classification of enriched KEGG terms in the DEGs in the RT vs RC comparison. (b)}

\section{Classification of enriched KEGG terms in the DEGs in the ST vs SC comparison.}

(a)

$$
\text { RT vs RC }
$$

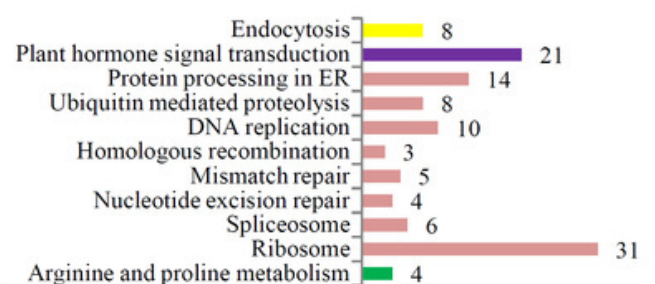

Arginine and proline metabolism $=4$

Cysteine and methionine metabolism _ 6

Glycine, serine and threonine metabolism 5

Phenylalanine, tyrosine and tryptophan_ 4

Flavonoid biosynthesis 7

Phenylpropanoid biosynthesis

Stibenoid, diarylheptanoid and gingerol $=4$

Amino sugar and nucleotide sugar metabolism - 6

Ascorbate and aldarate metabolism_ 6

Citrate cycle

Galactose metabolism $=3$

Glycolysis_ 12

Glyoxylate and dicarboxylate metabolism 3

Inositol phosphate metabolism_ 3

Pentose and glucuronate interconversions__ 11

Pyruvate metabolism_ 8

Starch and sucrose metabolism__ 14

Carbon fixation in photosynthetic organisms - 3

Biosynthesis of amino acids__ 15

Carbon metabolism__ 15

Biosynthesis of unsaturated fatty acids 7

Cutin, suberine and wax biosynthesis

Glycerolipid metabolism_ 4

Glycerphosphospholipid metabolism
Linoleic acid metabolism_

alpha-Linoleic acid metabolism_ 5

Porphyrin and chlorophyll metabolism_- 5

Terpenoid-quinone biosynthesis -4

Cyanoamino acid metabolism -3

Glutathione metabolism_ 4

beta-Alanine metabolism 5

Carotenoid biosynthesis_e 3

Limonene and pinene degradation $=3$

Purine metabolism__ 5

Circadian rhythm-plant-1

Plant-pathogen interaction
Fatty acid metabolism 9

Fatty acid elongation $=3$

Pyrimidine metabolism_- 58

$$
0
$$

20

401000

(b)

ST vs SC

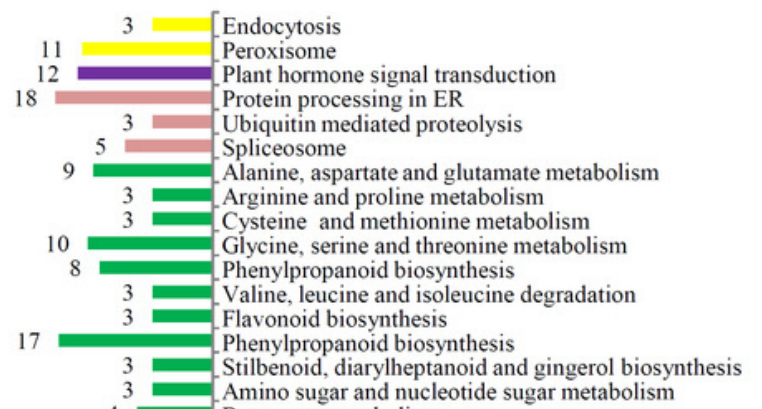

3 Amino sugar and nucleotide sugar metabolism

$4 \quad$ Butanoate metabolisin

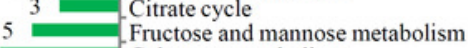

9 Galactose metabolism

8 Glycolysis

8 Glyoxylate and dicarboxylate metabolism

4 Inositol phosphate metabolism

3 Pentose and glucuronate intercongensis

6 Pentose phosphate pathway

21 Pyruvate metabolism

11 - Starch and sucrose metabolism

4 Sulfur metabolism

12 - 2-Oxocarboxylic acid metabolism

22

Biosynthesis of amino acids
Carbon metabolism

6 . Fatty acid metabolism

5 Biosynthesis of unsaturated fatty acids

4 Cutin, suberine and wax biosynthesis

5 Glycerophospholipid metabolism

5 Linolenic acid metabolism

3 Nicotinate and nicotinamide

$8 \quad$ Porphyrin and chlorophyll metabolism

3 Thiamine metabolism

4 Ubiquinone and other terpenoid

6 Cyanoamino acid metabolism

3 Glutathione metabolism

3 Taurine and hypotaurine metabolism

4 Alanine metabolism

5 Carotenoid biosynthesis

3 Diterpenoid biosynthesis

5 terpenoid backbone biosynthesis

4 - Circadian rhythm-plant

7 Plant-pathogen interaction

Cellular process Environmental information processing $100 \quad 10$

Metabolism

Organismal systems 
Figure 4

Identification and differential analysis of the hormonal network in cucumber

(A) KEGG pathway enrichment analysis of the DEGs showed the top 20 enriched KEGG terms.

Rich factor is the proportion of differentially expressed genes in all genes. (B-H) Overview of

various hormonal signaling network in cucumber. Red indicated the ST predominantly

expressed genes and green indicated the RT predominantly expressed genes.

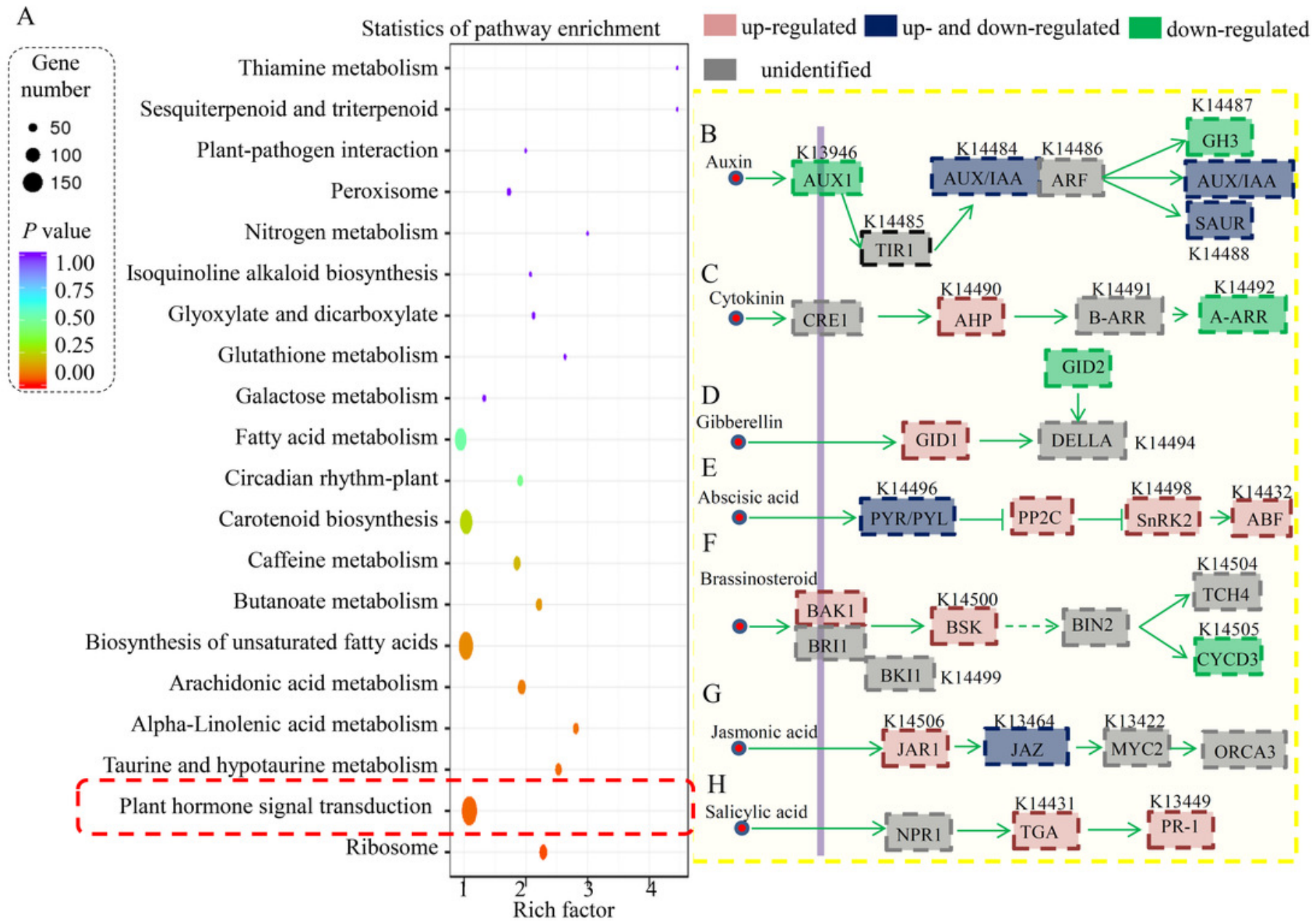




\section{Figure 5}

Identification of classical defense-related DEGs.

(A) Identification of differential expressed NBS genes between the resistant and susceptible cultivated varieties by $S$. fuliginea infection. (B) Identification of differential expressed glucanase genes between the resistant and susceptible cultivated varieties by $S$. fuliginea infection. (C) Identification of differential expressed chitinase genes between the resistant and susceptible cultivated varieties by $S$. fuliginea infection. (D) Identification of differential expressed peroxidase genes between the resistant and susceptible cultivated varieties by $S$. fuliginea infection. All the up-regulated genes were grouped into the Class I and all downregulated genes were grouped into the Class II.

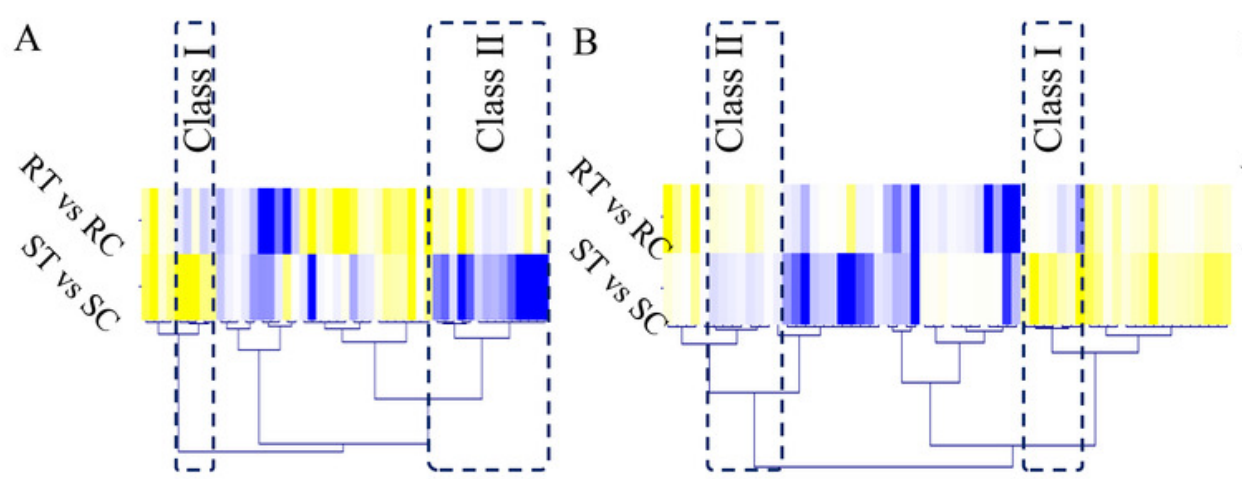

NBS genes
Glucanase genes

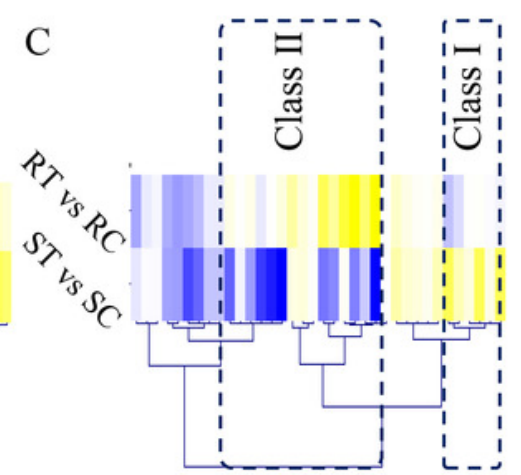

Chitinase genes

D
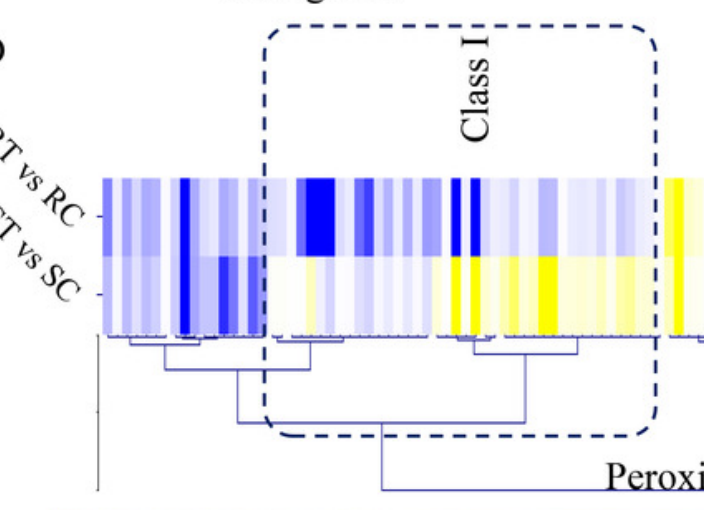

$-1.0$

0

Peroxidase genes

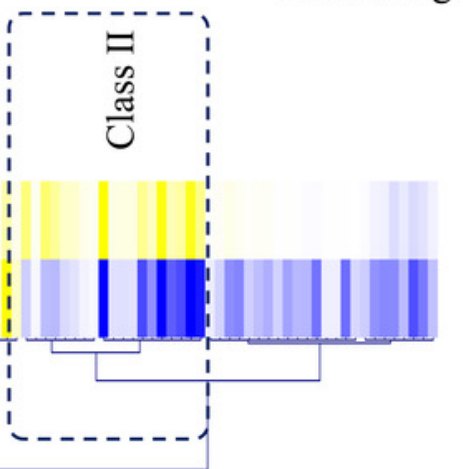


Figure 6

Validation of the expression levels of several key genes.

The relative expression levels of eight key genes under the $S$. fuliginea infection. Each bar shows the mean $\pm \mathrm{SD}(n=3)$ of triplicates. The significantly changes $(P<0.05)$ in expression levels of these genes between the treatments and control were indicated by "*”.
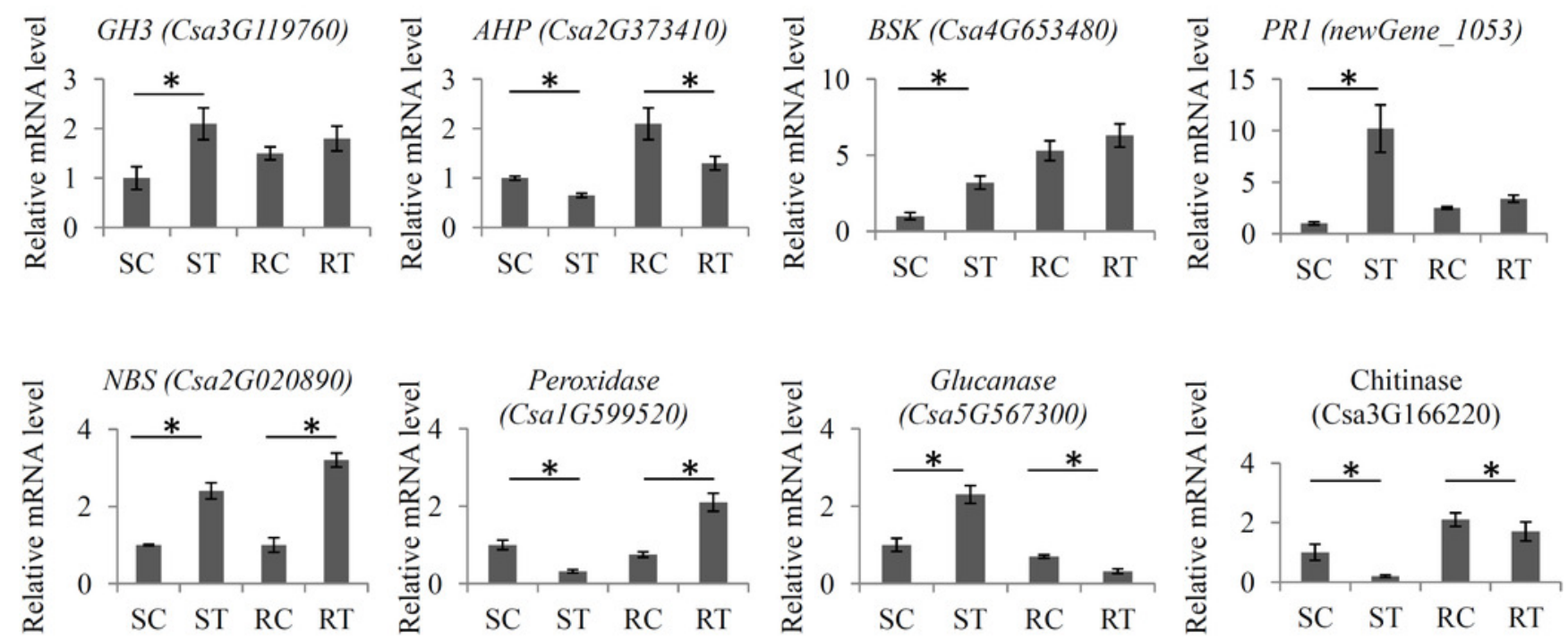